\title{
Bone Edema After Ankle Sprain and Update
}

\author{
M Ballester*1, G Lucar ${ }^{1}$ and Shahdad Saeedi ${ }^{2}$ \\ ${ }^{1}$ Consorci Sanitari del Maresme Hospital de Mataró, Barcelona, Spain \\ ${ }^{2}$ MedStar Georgetown University Hospital, Washington, US
}

Received: 阱January 23, 2019; Published: 制January 25, 2019

*Corresponding author: M Ballester, Consorci Sanitari del Maresme Hospital de Mataró, Barcelona, Spain

\section{Mini Review}

Acute ankle sprains are one of the most common musculoskeletal injuries. Studies in both the United States and European countries have shown that $30 \%$ of all athletic injuries involve ankle sprains [1]. Patients often present to their primary care physician or emergency department from the lateral ankle pain and instability after a plantarflexion inversion injury. A systematic review showed that at 1-year follow-up after conservative treatment, $5 \%$ to $33 \%$ of the patients still experience pain and instability, $34 \%$ of patients reported at least one recurrent sprain and 15\% to $64 \%$ reported incomplete recovery from their initial injury [2]. It is critical for us to note the associated injuries that may concomitantly exist with a lateral ankle sprain such as peroneal tendon tears, osteochondral lesions, intra-articular loose bodies or bone edema which may result in chronic symptoms.

Factors that may be associated with poor recovery in the shortterm include: the intensity of pain, difficulties weightbearing, restricted joint range of motion and the patient's functional ability [3]. With the increased popularity for obtaining an MRI post-ankle sprain, more bone edema or bone bruise is detected. This should not be overlooked as we have found strong clinical correlation to help us better treat this subgroup of patients. Bone bruise or bone marrow edema is a subchondral osseous fracture of the cancellous microarchitecture with accompanied local hemorrhage and edema [4]. The trabecular microarchitecture of the bone fails after an ankle sprain, and a compression fracture occurs. Bone bruise or bone marrow edema has to be differentiated from osteochondral lesions, in this case the subchondral bone plate is disrupted and the subchondral bone is exposed with or without a cartilage cap. The incidence of bone bruise after ankle sprain is variable, ranging from 7 to $40 \%$ in most cases, with bone bruise being associated with an ankle ligament rupture. In some instances, the bone marrow lesion level, intensity and location can take treatment precedence over the soft tissue injury [5]; making MRI an essential diagnostic tool in these situations. The diagnosis of bone marrow edema after sustaining an ankle sprain is made on a T2-weighted image which shows increased signal intensity and decreased signal intensity on a T1-weighted image. The location and pattern of the osseous injury has been studied by Labovitz being most frequently found in the medial talar dome.

With appropriate treatment following an ankle sprain the clinical prognosis of bone edema is usually favorable. The initial treatment algorithm after an ankle sprain can be superior to making the patient non-weightbearing and allowing them to bear weight to reduce joint stiffness. The actual evidence does not show that bone marrow edema after an ankle sprain is a prognostic factor for recovery [3]. Post-traumatic bone marrow edema that presents on MRI with concurrent symptomatic pain to the particular area for more than three months following trauma is a pathological state and a reason for treatment.

a) There is normalization of the bone edema on MRI which is usually seen within 6 to 12 months.

b) There is no evidence that would suggest this kind of lesion needs specific treatment.

c) It is important to note that the presence of a bone bruise should not delay rehabilitation, as one of the positive prognostic indicators is beginning proprioceptive training early.

It is also imperative to differentiate the bone marrow that has been injured of the subchondral plate. In these cases, one can do what is diagnostic for an OCD lesion and the treatment differs by reinforcing trabecular bone with a scaffold, to give the bone structural integrity to heal and give the cartilage a strong foundation. Being more conscious and analyzing the data we obtain in toto from an MRI study is vastly important and most often neglected. Advanced imaging can help paint a more precise and accurate picture of the root problem only if all aspects of the study are utilized and interpreted by the ordering physician as many times radiological reports are not all inclusive [6,7]. 


\section{References}

1. Saxena A, Luhadiya A, Ewen B, Goumas C (2011) Magnetic resonance imaging and incidental findings of lateral ankle pathologic features with asymptomatic ankles. J Foot Ankle Surg 50(4): 413-415.

2. Van Rijin RM, van Os AG, Bernsen RM, Luijsterburg PA, Koes BW, et al. (2008) What is the clinical course of acute ankle sprains? A systematic literature review. Am J Med 121(4): 324-331.

3. Thompson JY, Byrne C, Williams MA (2017) Prognostic factors for recovery following acute lateral ankle ligament sprain: A systematic review. BMC Musculoskelet Disord 18(1): 421.

ISSN: 2574-1241

DOI: 10.26717/BJSTR.2019.13.002435

M Ballester. Biomed J Sci \& Tech Res

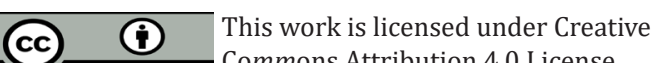

Submission Link: https://biomedres.us/submit-manuscript.php
4. Yao I, Lee JK (1988) Occult intraosseous fracture: Detection with MR imaging. Radiology 167(3): 749-751.

5. Rios AM, Rosenberg ZS, Bencardino JT, Rodrigo SP, Theran SG (2011) Bone marrow edema patterns in the ankle and hindfoot: Distinguishing mri features. Am J Roentgenol 197(4): 720-729.

6. Labovitz JM, Schweitzer ME (1998) Occult osseous injuries after ankle sprains: Incidence, location, pattern and age. Foot Ankle Int 19(10): 661667.

7. Longo UG, Loppini M, Romeo G, van Dijk CN (2013) Bone bruises associated with acute ankle ligament injury: Do they need treatment? Knee Surg Sports Traumatol Arthrosc 21(6): 1261-1268.

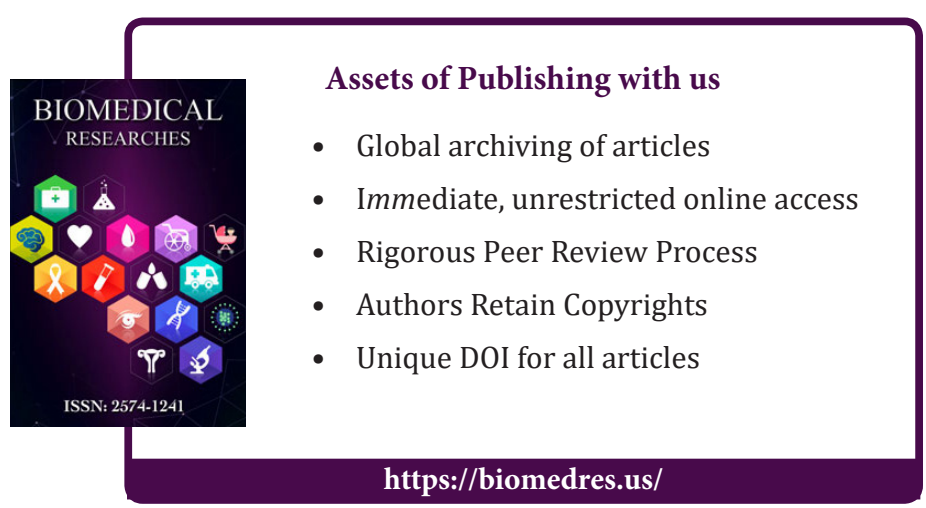

